Since $S_{1} T=c^{-1} b^{-1} c b c$ is the transform of $c$ by $b c$, it is of period three.

The final relation (10) becomes

$$
\begin{aligned}
\left(b c^{-1} b^{-1} c \cdot b^{-1} c b c\right)^{2} & =\left(c^{-1} b c b^{-1} \cdot b^{-1} c b c\right)^{2}=\left(c^{-1} b c b^{2} c b c\right)^{2} \\
& =c^{-1} b\left(c b^{2}\right)^{4} b^{-1} c=I .
\end{aligned}
$$

Since $S_{j}$ is commutative with $S_{1}$, the condition $S_{j}^{3}=I$ follows from $\left(b^{-1} c^{-1} b^{2} c^{-1}\right)^{3}=I$ or $\left(c b^{2} c b\right)^{3}=I$.

The University of Chicago, December 11, 1902 .

\title{
NOTE ON A PROPERTY OF THE CONIC SECTIONS.
}

BY PROFESSOR H. F. BLICHFELDT.

(Read before the San Francisco Section of the American Mathematical Society, December 20, 1902. )

IT is easily proved that if $P, Q, R$ are any three points on the conic $A x^{2}+B y^{2}=1$, and $O$ the center of the conic, then the areas of the triangles $O P Q, O P R, O Q R$ will satisfy an equation independent of the position of the points $P, Q, R$. If $a, b, c$ are the areas in question, this equation is

(1) $a^{4}+b^{4}+c^{4}-2 a^{2} b^{2}-2 a^{2} c^{2}-2 b^{2} c^{2}+16 A B a^{2} b^{2} c^{2}=0$.

Now we can prove that such an invariant relation is possible for no plane curves except the central conics; $i$. e., if we seek $a$ plane curve $C$ and a point $O$ in its plane such that, if $P, Q, R$ are any three points on $C$, the triangles $O Q R$, ORP, OPQ are connected by a relation independent of the coördinates of the points $P, Q, R$, we find $C$ to be a central conic section and $O$ its center.

To prove this theorem, let $O$ be the origin of coördinates, and let the coördinates of $P, Q, R$ be respectively $x_{1}, y_{1} ; x_{2}, y_{2}$; $x_{3}, y_{3}$. Then twice the areas of the three triangles are

$$
\begin{gathered}
2 a= \pm\left(r_{2} x_{3}-y_{3}^{\prime} x_{2}\right), \quad 2 b= \pm\left(y_{3} x_{1}-y_{1} x_{3}\right) \\
2 c= \pm\left(y_{1} x_{2}-y_{2} x_{1}\right)
\end{gathered}
$$


which expressions are functions of the three independent variables $x_{1}, x_{2}, x_{3} ; y$ being considered a given function of $x$ for points on the curve.

As $a, b, c$ must satisfy a relation independent of $x_{1}, x_{2}, x_{3}$, the Jacobian $\partial(a, b, c) / \partial\left(x_{1}, x_{2}, x_{3}\right)$ must vanish. If $y_{1}^{\prime}$ represents $d y_{1} / d x_{1}$, etc., we find

$$
\begin{gathered}
y_{3}^{\prime}\left\{y_{3}\left[x_{1} y_{2}-x_{2} y_{1}+x_{1} x_{2}\left(y_{2}^{\prime}-y_{1}^{\prime}\right)\right]+x_{3}\left(x_{2} y_{1} y_{1}^{\prime}-x_{1} y_{2} y_{2}^{\prime}\right)\right\} \\
+x_{3}\left[\left(x_{1} y_{2}-x_{2} y_{1}\right) y_{1}^{\prime} y_{2}^{\prime}+y_{1} y_{2}\left(y_{2}^{\prime}-y_{1}^{\prime}\right)\right]+y_{3}\left(x_{2} y_{1} y_{1}^{\prime}-x_{1} y_{2} y_{2}^{\prime}\right)=0,
\end{gathered}
$$

$$
y_{3}^{\prime}\left(y_{3} k+x_{3} l\right)+x_{3} m+y_{3} l=0,
$$

$k, l, m$ being functions of $x_{1}, x_{2}$ only, and therefore independent of $x_{3}$.

Two cases $(a)$ and $(\beta)$ may now present themselves as follows :

(a) The functions $k, l, m$ are not all identically zero. In this case the equation (2) gives, when integrated,

$$
y_{3}^{2} k+2 y_{3} x_{3} l+x_{3}^{2} m=f\left(x_{1}, x_{2}\right) .
$$

If we give to $x_{1}$ and $x_{2}$ arbitrary constant values, the equation (3) represents a conic section with its center at $O$.

$(\beta)$ The functions $k, l, m$, are all zero. We must then have $x_{2} y_{1}^{\prime}-y_{2}=0$. Giving to $y_{1}^{\prime}$ a definite constant value, we obtain the equation of a straight line - a special case of (3).

The theorem stated above is therefore proved.

It may be noticed that $f\left(x_{1}, x_{2}\right)$ in (3) may be multiple valued. The equation will then represent a series of similar conics similarly placed. If these are finite in number, say $n$, we find that, if $P, Q, R$ be located anywhere on this system of curves, the areas $a, b, c$ of the three triangles considered will satisfy an equation of degree

$$
6 n+18 n(n-1)+6 n(n-1)(n-2)
$$

at most, whose left-hand member is composed of factors of form similar to (1), as the reader may prove without much difficulty. 